\title{
Ex Post Pricing in the Co-Optimized Energy and Reserve Market
}

\author{
Tongxin Zheng, Member, IEEE, and Eugene Litvinov, Member, IEEE
}

\begin{abstract}
This paper discusses an alternative to the ex post pricing model currently under development within ISO New England's (ISO NE) ancillary service market. Since ISO NE's real-time ancillary service market co-optimizes both energy and reserve products, ex post pricing is needed for both energy and reserve. A co-optimization approach for ex post pricing requires defining complex pricing rules and implementing heuristics to ensure consistent binding constraints with the ex ante dispatch. In order to avoid complexity and uncertainty of required heuristics, a new ex post pricing schema is proposed to calculate the ex post energy and reserve prices in a decoupled way while recognizing the coupling effect of energy and reserve. In the proposed approach, ex post energy prices are first computed by incorporating the ex ante marginal opportunity cost of reserve in the energy offer; the ex post reserve prices are then calculated by considering the ex post marginal opportunity cost of energy. This approach simplifies the implementation of ex post pricing rules in the ISO NE market. Numerical examples are presented to further demonstrate the validity of this approach.
\end{abstract}

Index Terms-Electricity market, ex ante pricing, ex post pricing, locational marginal pricing, opportunity cost, optimization, power systems.

\section{NOMENCLATURE}

Index for all generators.

Index for all price-sensitive demands. Index of set $\{$ any $i$ or $j\}$.

Index for all reserve categories, including 10-min spinning $(s p)$, 10-min non-spinning $(n s p)$, and 30-min operating $(o p)$ reserves.

$k \quad$ Index for all transmission constraints.

$l \quad$ Index for all local reserve constraints.

$s \quad$ Index for all categories of system-wide reserve constraints.

$n \quad$ Index for all buses in the system.

$G_{\text {on }} \quad$ Set of the online generators.

$C_{R} \quad$ Set of reserve categories, $\{s p, n s p, o p\}$.

$F_{g}$ or $d \quad$ Set of flexible generators $(g)$ or loads $(d)$.

$B_{g}$ or $d \quad$ Set of generators $(g)$ or loads $(d)$ with full capacity cleared in the ex ante dispatch.

$T_{i}$ or $j$

$H_{t, i}$ or $j$

\section{$l r$}

$\min$

$\max$

expost Set of reserves categories with positive marginal opportunity cost of reserve and nonzero MW of ex post reserve quantity for generator $i$ or load $j$.

Manuscript received March 17, 2006; revised May 12, 2006. Paper no. TPWRS-00143-2006.

The authors are with ISO New England Inc., Holyoke, MA 01040 USA (e-mail: tzheng@iso-ne.com; elitvinov@iso-ne.com).

Digital Object Identifier 10.1109/TPWRS.2006.882457 $s r$

ex ante

$S E$

10

$P_{\text {loss }}$

$\delta_{t, i}^{x}$ or $j$

Set of reserve categories that have greater marginal opportunity cost of reserve than that of type $t$ for generator $i$ or load $j$.

Superscript that indicates the local reserve.

Superscript that indicates the system-wide reserve.

Superscript that indicates the minimum value.

Superscript that indicates the maximum value.

Superscript that indicates a value obtained from the ex post calculation.

Superscript that indicates a value obtained from the ex ante dispatch.

Superscript that indicates a value obtained from the state estimator, representing the current state.

Superscript or subscript that indicates a value that can be achieved in $10 \mathrm{~min}$.

Superscript or subscript that indicates a value that can be achieved in $30 \mathrm{~min}$.

Superscript that indicates the upper bound.

Superscript that indicates the lower bound.

Offer price for generator or penalty price.

Bid price for a price sensitive demand.

Offer price for reserve.

Reserve quantity or designation.

Generation output.

Consumption of the price sensitivity demand.

Fixed demand.

Slack variable or reserve deficit.

Active power flow over a transmission line.

Active power flow over an interface.

Amount of local or system-wide contingency or reserve requirement.

System active power losses.

Binary value that is 1 when the type $t$ reserve from generator $i$ or load $j$ belongs to a set $x$ (i.e., local reserve zone $l$, system-wide reserve $s$, or 10-min capability).

$R C_{i}^{D I} \quad$ Ramping capability of unit $i$ within the dispatch interval $D I$.

$R C \quad$ Ramp capability for a generator or load.

oc Marginal opportunity cost for a resource.

$\Delta \quad$ Small positive perturbation. 


\section{INTRODUCTION}

L OCATIONAL marginal pricing (LMP) [1] has been adopted by the majority of electricity markets in the United States. Typical electricity markets are composed of both day-ahead and real-time markets. Spot electricity prices are calculated in the real-time market. However, there are two ways of determining the real-time electricity prices: one is "to set the prices using near real-time estimates (ex ante)" [2]; the other is to set the prices "based on the prices of the actual marginal resource clearing the market in real time (ex post)" [2].

Due to the non-storable nature of electricity, dispatching generating resources to meet the real-time load in an economic and reliable way is a very important task of the market operator. Therefore, in the market environment, the spot electricity prices should provide sufficient incentives for all the dispatchable resources to follow dispatch instructions. Both ex ante and ex post prices combined with proper pricing rules can achieve this goal. NY ISO uses ex ante prices as the real-time prices and penalizes non-performing resources on the basis of reduced generation quantity [3], while ISO NE and PJM adopt the ex post pricing that provides dispatch incentives on the ground of rational prices [4], [5]. Compared with the ex ante pricing, the ex post pricing "creates incentives for bidders to act consistently with their bids" [2]. Based on this economic justification, we focus on the ex post pricing in this paper.

Co-optimization of energy and reserve products in real-time dispatch and pricing has been discussed extensively in the literature [6]-[10]. For instance, [6] models reserves by simulating contingency events; [7] proposes a zonal reserve model; while [8]-[10] consider the system-wide reserve requirements only. However, none of them is used for ex post pricing. Currently, there are two major approaches for ex post energy pricing. One starts from the dual problem of the ex ante dispatch problem [11], [12] and formulates the ex post pricing as a linear programming problem to find a set of prices that can reflect the actual behavior of generating resources. However, this method assumes that resources' actual performances are optimal, which is almost never true in real-time operations. In addition, the objective of the pricing problem (maximize or minimize the congestion revenue) is not well defined or justified.

Another approach, which has been adopted by PJM and ISO NE [13], uses an incremental optimization of the ex ante dispatch problem according to resources' actual operating conditions. At the same time, pricing rules are also established to ensure that the ex post pricing takes generating resources' performance into consideration. In practice, this works well, although it uses some empirical formulae. However, this approach is based on the energy-only market and does not explicitly consider the coupling effect between prices of ancillary services products and those of energy.

An incremental co-optimization seems to be a natural step for the ex post pricing of energy and reserve since ISO NE and PJM have been using the energy-only ex post pricing schema. However, this technique requires defining pricing rules based on the resource's ex ante dispatch status, which can be very complex due to the combinatorial nature of different binding statuses of resource level constraints. For instance, a generating unit with ramp, capacity, regulation, 10-min ramp, and 30-min ramp constraints can have at least 32 different states corresponding to the different binding statuses of these constraints. Each state may require a unique rule in the ex post pricing. In addition, heuristics have to be implemented to ensure similar binding statuses of constraints as those in the ex ante dispatch. This introduces uncertainties in the implementation.

The study of the co-optimized ex ante dispatch problem indicates that the coupling between energy and reserve products is the marginal opportunity cost of the dispatchable resource ([9] and [10] considered the total opportunity cost rather than the marginal opportunity cost in the optimization, and the pricing of reserve products does not include the effect of the marginal opportunity cost.). Recognizing the coupling effect of energy and reserve prices, as well as the limitations of the incremental co-optimization technique, we, therefore, propose to price energy and reserve in a decoupled fashion while properly taking into account the marginal opportunity cost. Not only does this reduce the uncertainty of co-optimization due to the introduced heuristics, but it also makes the pricing rules much simpler.

Since the ex post pricing is dependent on the ex ante dispatch and market rules, in this paper, we will first discuss the ex ante problem in Section II and the pricing principles in Section III. The proposed ex post pricing schema is presented in Section IV, followed by the numerical examples in Section V. Finally, the conclusion is drawn in Section VI.

\section{Ex ANTE Co-OptiMIZATION}

In general, an ex post pricing is based on an ex ante dispatch, and different ex ante dispatch formulations will result in different ex post pricing algorithms. In this paper, we use ISO NE's real-time co-optimization model as the base of the proposed ex post pricing method and discuss it in this section.

In the ISO NE market, the real-time dispatch problem is formulated as a linear programming problem with the objective to maximize the social surplus, subject to real-time operating constraints and the physical characteristics of resources. Under the co-optimized energy and reserve market [14], system-wide and locational reserve constraints are enforced for the market operator to procure enough reserves to cover the first and the second contingency events. Although [14] does not allow availability bids for reserve, we include availability bids for the purpose of generality. In short, the ex ante dispatch problem is formulated as

$$
\begin{aligned}
& \underset{P, R, L, S}{\operatorname{Min}} \sum_{i \in G_{o n}}\left(c_{i} \cdot P_{i}+\sum_{t \in C_{R}}\left(o_{t, i} \cdot R_{t, i}\right)\right) \\
& \quad+\sum_{j}\left(-b_{j} \cdot L_{j}+\sum_{t \in C_{R}}\left(o_{t, j} \cdot R_{t, j}\right)\right)+\sum_{l}\left(c_{l}^{l r} \cdot S_{l}^{l r}\right) \\
& \quad+\sum_{s}\left(c_{s}^{s r} \cdot S_{s}^{s r}\right)
\end{aligned}
$$

subject to the following constraints:

1) Energy Balance Constraint

$$
(\lambda): \sum_{i \in G_{\text {on }}} P_{i}=\sum_{j} L_{j}+P_{\text {loss }}+\sum_{n} D_{n} .
$$


Based on the base-case scenario, system losses can be linearized as a function of each individual resource's output

$$
\begin{aligned}
P_{\text {loss }}=\sum_{i}\left(\frac{\partial P_{\text {loss }}}{\partial P_{i}} \cdot P_{i}\right)+ & \sum_{j}\left(\frac{\partial P_{\text {loss }}}{\partial L_{j}} \cdot L_{j}\right) \\
& +\sum_{n}\left(\frac{\partial P_{\text {loss }}}{\partial D_{n}} \cdot D_{n}\right)+\text { offset }
\end{aligned}
$$

where offset is the linearization error. More details can be found in [15].

2) Transmission Constraints

$$
\left(\mu_{k} \leq 0\right): \text { flow }_{k} \leq \text { flow }_{k}^{\max } \text { for any } k .
$$

Same as system losses, the transmission flow is represented by a linear function of each individual resource's output

$$
\begin{aligned}
\text { flow }_{k}=\sum_{i}\left(\frac{\partial \text { flow }_{k}}{\partial P_{i}} \cdot P_{i}\right)+ & \sum_{j}\left(\frac{\partial \text { flow }_{k}}{\partial L_{j}} \cdot L_{j}\right) \\
& +\sum_{n}\left(\frac{\partial \text { flow }_{k}}{\partial D_{n}} \cdot D_{n}\right)+\text { bias }
\end{aligned}
$$

where bias is the linearization error.

3) Locational Reserve Constraints

$$
\begin{array}{r}
\left(\alpha_{l} \geq 0\right): \sum_{m=i, j} \sum_{t \in C_{R}}\left(\delta_{t, m}^{l} \cdot R_{t, m}\right)+\left(\text { Iflow }_{l}^{\max }-\text { Iflow }_{l}\right)+ \\
S_{l}^{l r} \geq Q_{l} \text { for any local reserve constraint } l .
\end{array}
$$

In the ISO NE market, a locational reserve constraint is used to cover the second contingency event caused by the loss of a generator or a second line in a local area. Therefore, the unloaded tie-line capacity, as well as the reserve provided by units located in the local reserve zone, can be utilized to cover the local contingency or reserve requirement within $30 \mathrm{~min}$. Note that the interface flow $\mathrm{Iflow}_{l}$ is a function of network injections, which can be calculated the same way as flow . $_{\text {. }}$

4) System-Wide Reserve Constraints

$$
\begin{aligned}
\left(\beta_{s} \geq 0\right): \sum_{\substack{m=i, j \\
\text { for any system-wide reserve constraint } s .}}\left(\delta_{t, m}^{s} \cdot R_{t, i}\right)+S_{s}^{s r} \geq Q_{s} \\
\quad
\end{aligned}
$$

5) Capacity Constraints for Online Units

$$
\left(\gamma_{i} \leq 0\right): P_{i}+\sum_{t \in C_{R}}\left(R_{t, i}\right) \leq P_{i}^{\max } \text { for any } i \in G_{\mathrm{on}} .
$$

6) Reserve Capacity Constraints for Dispatchable Loads

$$
\left(\gamma_{j} \leq 0\right): \sum_{t \in C_{R}}\left(R_{t, j}\right) \leq L_{j}-L_{j}^{\text {min }} \text { for any } j .
$$

7) Ramp Constraints for Online Units

$$
\begin{aligned}
& \left(\eta_{i}^{u p} \leq 0\right): P_{i} \leq P_{i}^{S E}+R C_{i}^{D I} \text { for any } i \in G_{\mathrm{on}} \\
& \left(\eta_{i}^{d n} \geq 0\right): P_{i} \geq P_{i}^{S E}-R C_{i}^{D I} \text { for any } i \in G_{\mathrm{on}} .
\end{aligned}
$$

8) 10-min Ramp Capability Constraints

$$
\begin{aligned}
& \left(\eta_{i}^{10} \leq 0\right): \sum_{t \in C_{R}}\left(\delta_{t, i}^{10} \cdot R_{t, i}\right) \leq R C_{i}^{10} \text { for any } i \\
& \left(\eta_{j}^{10} \leq 0\right): \sum_{t \in C_{R}}\left(\delta_{t, j}^{10} \cdot R_{t, j}\right) \leq R C_{j}^{10} \text { for any } j .
\end{aligned}
$$

9) 30-min Ramp Capability Constraints

$$
\begin{aligned}
& \left(\eta_{i}^{30} \leq 0\right): \sum_{t \in C_{R}}\left(R_{t, i}\right) \leq R C_{i}^{30} \text { for any } i \\
& \left(\eta_{j}^{30} \leq 0\right): \sum_{t \in C_{R}}\left(R_{t, j}\right) \leq R C_{j}^{30} \text { for any } j .
\end{aligned}
$$

10) Upper and Lower Bounds of Control Variables

$$
\begin{aligned}
& \left(\eta_{i}^{\text {min }} \geq 0\right): P_{i} \geq P_{i}^{\min } \text { for any } i \in G_{o n} \\
& \left(\eta_{j}^{\text {max }} \leq 0\right): L_{j} \leq L_{j}^{\text {max }} \text { for any } j \\
& \left(\eta_{j}^{\text {min }} \geq 0\right): L_{j} \geq L_{j}^{\text {min }} \text { for any } j \\
& \left(\xi_{t, i}^{\text {min }} \geq 0\right): R_{t, i} \geq 0 \text { for any } i \text { and } t \in C_{R} \\
& \left(\xi_{t, j}^{\text {min }} \geq 0\right): R_{t, j} \geq 0 \text { for any } j \text { and } t \in C_{R} \\
& \left(\rho_{l}^{\text {min }} \geq 0\right): S_{l}^{l r} \geq 0 \text { for any } l \\
& \left(\rho_{s}^{\text {min }} \geq 0\right): S_{s}^{s r} \geq 0 \text { for any } s
\end{aligned}
$$

where $\lambda, \mu, \alpha, \beta, \gamma, \eta, \xi$, and $\rho$ are the shadow prices for constraints (1.1)-(1.19), respectively. The decision variables are the generation MW $P_{i}$, the dispatchable load consumption $L_{j}$, reserve designations $R_{t, i}$ or ${ }_{j}$, and the reserve deficits $S$. In the above formulation, a single-block offer curve per resource is assumed, which can be easily extended to multiblock offers. In addition, the reserves from offline units are not considered here for simplification, since they do not incur the opportunity cost in general.

Based on the marginal pricing concept, the ex ante energy price or $L M P$ for each bus $n$ and the reserve clearing price $(R C P)$ of each reserve product for each resource are defined as

$$
\begin{aligned}
L M P_{n}^{\text {ex ante }}= & \lambda\left(1+\frac{\partial P_{\text {loss }}}{\partial D_{n}}\right)-\sum_{k}\left[\mu_{k} \cdot \frac{\partial \text { flow }_{k}}{\partial D_{n}}\right] \\
& +\sum_{l}\left[\alpha_{l} \cdot \frac{\partial I f l o w_{l}}{\partial D_{n}}\right] \\
R C P_{t, i}^{\text {ex ante }}= & \sum_{l}\left[\alpha_{l} \cdot \delta_{t, i}^{l}\right]+\sum_{s}\left[\beta_{s} \cdot \delta_{t, i}^{s}\right] .
\end{aligned}
$$

Under the optimal conditions, the following relationships can be obtained from Karush-Kuhn-Tucker (KKT) conditions:

$$
\begin{aligned}
L M P_{i}^{e x} \text { ante } & =c_{i}-\gamma_{i}-\eta_{i}^{u p}-\eta_{i}^{d n}-\eta_{i}^{\text {min }} \\
R C P_{t, i}^{\text {ex ante }} & =o_{t, i}-\gamma_{i}-\eta_{i}^{l 0} \cdot \delta_{t, i}^{10}-\eta_{i}^{30}-\xi_{t, i}^{\text {min }} \\
L M P_{j}^{\text {ex ante }} & =b_{j}-\gamma_{j}-\eta_{j}^{\text {max }}-\eta_{j}^{\text {min }} \\
R C P_{t, j}^{\text {ex ante }} & =o_{t, j}-\gamma_{j}-\eta_{j}^{l 0} \cdot \delta_{t, j}^{10}-\eta_{j}^{30}-\xi_{t, j}^{\text {min }} .
\end{aligned}
$$

(3.1) and (3.2) indicate that the energy price $L M P_{i}^{e x}$ ante is coupled with the reserve price $R C P_{t, i}^{e x}$ ante for an online generator $i$ when the capacity constraint $(1.5)$ binds $\left(\gamma_{i}<0\right)$. Especially, when the reserve dispatch is not constrained (i.e., (1.9) 
and (1.11) are not binding), $-\gamma_{i}$ is equal to $R C P_{t, i}^{e x}$ ante $-o_{t, i}$ representing the marginal opportunity cost of reserve; when the energy dispatch is not constrained (i.e., (1.7), (1.8), and (1.13) are not binding), $-\gamma_{i}$ is equal to $L M P_{i}^{e x}$ ante $-c_{i}$, representing the marginal opportunity cost of energy. However, when (1.5) does not bind $\left(\gamma_{i}=0\right)$, the energy price and the reserve price are independent of each other for generator $i$. The energy and reserve prices for a dispatchable load have a similar relationship.

\section{Ex Post PRICING PRINCIPLES}

As discussed in Section I, the intent of ex post pricing is to encourage resources to follow the system operator's dispatch instructions. However, there may be many different ways to achieve it according to market rules established in different electricity markets. In order to come up with a good design of ex post pricing in the co-optimized reserve market, we need to first define the pricing principles and rules that should be followed in the ex post pricing. Those rules and principles should not only provide guidance for the design of ex post pricing schema but also serve as the criteria for evaluating ex post prices. Pricing principles are discussed as follows.

1) The goal of ex post pricing is not to re-dispatch the system based on the actual operating condition; instead, it should produce rational prices to all the resources according to their actual performance. A re-dispatch will result in a set of optimal prices; however, these prices would not reflect what happened in the real world.

2) Ex post prices should reflect the coupling effect between energy and reserve products and preserve the pricing formulation established in the ex ante dispatch. This ensures consistency between the ex ante dispatch and the ex post pricing, since the dispatchable resources will generally respond to the ex ante prices and dispatches.

3) Ex post prices should be a set of prices that encourage resources to follow their dispatch instructions.

3.1) Dispatchable resources should follow their dispatch instructions, which are generated by the ex ante dispatch. Although the ex ante dispatch instructions are indicative, in a perfect world, the ex post prices should be the same as ex ante prices if all the dispatchable resources obey the ex ante dispatch. Otherwise, there will be no incentive for the resources to follow dispatch instructions.

3.2) Rules must be established to evaluate the performance of resources. Non-performing resources should be penalized on the basis of their ex post prices. They should either pay a penalty or become price takers in the ex post pricing. The ex post prices should be the best prices possible for units that properly follow their dispatch instructions. That is ex post prices should be able to satisfy all properly performing units according to their physical characteristics.

3.3) The real-time offer price of a dispatchable generator in the ex post pricing should not exceed its ex ante energy price, even if the unit correctly follows its dispatch instruction. This attempts to prevent generators from over-generating.

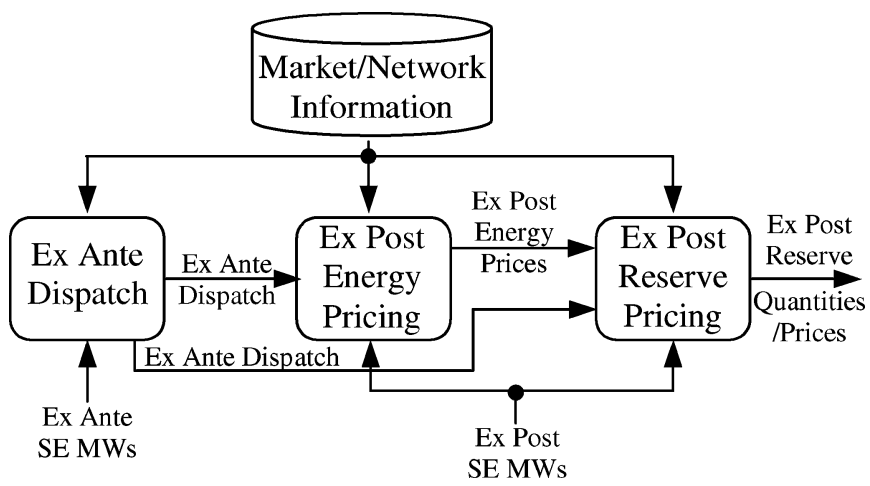

Fig. 1. Ex post pricing procedure.

3.4) The real-time willing-to-pay price of a dispatchable load in the ex post pricing should not be lower than its ex ante energy price, even if the load correctly follows its dispatch instruction. This is intended to prevent dispatchable loads from over-consuming.

3.5) The ex post reserve price and quantity of a dispatchable resource should not exceed its ex ante reserve prices and reserve quantities, respectively. This prevents the dispatchable resource from overperforming in the reserve market.

Different rules of ex post pricing will result in different implementations. In the next section, we will discuss the proposed ex post pricing mechanism based on the above pricing principles.

\section{Proposed Ex Post Pricing Schema}

The analysis of the ex ante price relationship in Section II shows that the energy price is coupled with reserve prices through the shadow prices of capacity constraints (1.5), (1.6). In addition, the reserve clearing prices are determined by the reserve offer price and the marginal opportunity cost of energy, which is the difference between the energy price and the offer price of each dispatchable resource under normal conditions or the reserve penalty under reserve scarcity condition. The above observations suggest that we could price energy and reserve separately while respecting the coupling effect between them. Since the coupling effect between energy and reserve prices is the marginal opportunity cost, we propose the following procedure for ex post pricing.

1) Calculate the ex post energy prices with respect to the energy-only constraints. In the energy pricing, the ex ante marginal opportunity cost of reserve, which is the difference between the reserve clearing price and its reserve offer price of the dispatchable resource, is added to the energy offer price.

2) Calculate the ex post reserve prices to meet the actual reserve available with respect to reserve-only constraints. In the reserve pricing, the ex post marginal opportunity cost of energy, which is the difference between the ex post energy price and the offer price of a dispatchable resource, is considered as the cost of reserve in addition to the reserve offer price.

Fig. 1 shows the data flow between the ex ante dispatch and the ex post pricing. In the ex ante dispatch, the dispatch decisions are made based on the market and power system informa- 
tion and the initial output level of each resource (SE MW). The ex ante dispatch results (generation and consumption schedules, reserve designations, ex ante LMPs and reserve clearing prices, and necessary binding status of constraints), as well as the actual performance data (ex post SE MW), are sent to the ex post energy pricing for the calculation of ex post energy prices. Further, the ex post reserve clearing prices and MW quantities are computed using ex post energy prices (LMPs), ex ante reserve clearing prices and quantities, ex post SE MWs, and market/network information.

In the following, we will discuss each pricing algorithm in more detail.

\section{A. Ex Post Energy Pricing}

Based on the second pricing principle stated in Section III, the ex post energy pricing problem should produce the energy prices defined in (2.1). However, (2.1) is derived from the energy-reserve co-optimization, and therefore, the full ex ante problem formulation cannot be used. Since the goal of ex post energy pricing is to find the energy prices assuming the actual reserve and its cost from each resource is given, we can use the ex ante formulation while removing the reserve-related constraints. Nevertheless, this does not mean that the energy prices do not reflect the coupling effect of reserves.

When the energy and reserves are decoupled in the pricing, the ex ante marginal opportunity cost of reserve for different type of resources (loads and generators) must be considered in the ex post energy pricing as follows.

1) According to the ex ante dispatch analysis, to produce one more MW of energy, a unit with full capacity cleared (constraint (1.5) binds) must sacrifice one MW of reserve, and therefore, the all-in cost for this incremental MW of energy is the sum of its energy offer price and its lost profit from reserve market. A unit with capacity partially cleared (constraint (1.5) does not bind) will not give up its reserve profit from the incremental energy, and therefore, its all-in cost is its offer price.

2) The situation of a dispatchable load that provides reserve is similar to the generator. For any price sensitive load that provides reserves with constraint (1.6) binding, reducing one more MW of energy consumption not only foregoes the benefit of consuming energy but also loses one more MW of reserve profit. The dispatchable load that provides reserve with non-binding constraint (1.6) does not receive additional benefit from reserve by decreasing or increasing its consumption level.

Combining the above analysis with the incremental optimization technique, we can formulate the ex post energy pricing problem as follows:

$$
\underset{P, S, L}{\operatorname{Min}} \sum_{i}\left(c_{i}^{\text {ex post }} \cdot P_{i}\right)+\sum_{l}\left(c_{l}^{l r} \cdot S_{l}^{l r}\right)-\sum_{j}\left(b_{j}^{\text {ex post }} \cdot L_{j}\right)
$$

S.T.

\section{1) Incremental Energy Balance}

$\left(\lambda^{\text {ex post }}\right): \sum_{i}\left(P_{i}-P_{i}^{S E}\right)-\sum_{j}\left(L_{j}-L_{j}^{S E}\right)=P_{\mathrm{loss}}-P_{\mathrm{loss}}^{S E}$.
2) Ex Ante Binding Transmission Constraints

$$
\begin{aligned}
\left(\mu_{k}^{\text {ex post }} \leq 0\right) & : \text { flow }_{k} \leq \text { flow }_{k}^{S E} \text { for any } \\
& \text { ex ante binding transmission constraint } k .
\end{aligned}
$$

3) Ex Ante Binding Local Reserve Constraints

$$
\begin{array}{r}
\left(\alpha_{l}^{\text {energy }} \geq 0\right):- \text { Iflow }_{l}+S_{l}^{l r} \geq S_{l}^{l r, \text { ex ante }}-\text { Iflow }_{l}^{S E} \\
\text { for any ex ante binding constraint } l .
\end{array}
$$

4) Boundary for Dispatchable Units

$$
\begin{cases}P_{i}^{S E}-\Delta_{d n} \leq P_{i} \leq P_{i}^{S E}+\Delta_{\mathrm{up}}, & \text { if } i \in F_{g} \\ P_{i}=P_{i}^{S E}, & \text { if } i \notin F_{g} \\ & \text { for any } i .\end{cases}
$$

5) Boundary for Dispatchable Loads

$$
\begin{cases}L_{j}^{S E}-\Delta_{d n} \leq L_{j} \leq L_{j}^{S E}+\Delta_{\text {up }}, & \text { if } j \in F_{d} \\ L_{j}=L_{j}^{S E}, & \text { if } j \notin F_{d} \\ & \text { for any } j\end{cases}
$$

where

$F_{g}=\left\{\right.$ any $i$, where $P_{i}^{S E} \leq$ defines the set of online $1.1 \cdot P_{i}^{e x}$ ante $\} \quad$ dispatchable generators that perform properly and are eligible to set ex post energy prices;

$F_{d}=\left\{\right.$ any $j$, where $L_{j}^{S E} \geq$ defines the set of online $0.9 \cdot L_{j}^{e x}$ ante $\} \quad$ dispatchable loads that perform correctly and are eligible to set ex post energy prices;

$P_{\text {loss }}$, flow $k$, and Iflow ${ }_{l}$ are each a function of $P_{i}, L_{j}$, and $D_{n}$.

$F_{g}$ and $F_{d}$ present the performance metrics of dispatchable resources in the ISO NE market, and different market rules will result in different implementations of flexible resources in the ex post pricing.

According to the analysis of the ex post energy prices and pricing rules 3.3 and 3.4 , we can define the energy offer price for the dispatchable generator $i$ as

$$
\begin{aligned}
& c_{i}^{\text {ex }} \text { post } \\
& \quad= \begin{cases}\min \left(L M P_{i}^{\text {ex ante }}, c_{i}^{S E}+o c_{i}^{\text {reserve }}\right), & \text { if } i \in F_{g} \\
0, & \text { if } i \notin F_{g}\end{cases}
\end{aligned}
$$

where

$$
\begin{aligned}
& o c_{i}^{\text {reserve }}= \begin{cases}\min _{t \in T_{i}}\left(R C P_{t, i}^{e x} \text { ante }-o_{t, i}\right), & \text { for } i \in B_{g} \\
0, & \text { otherwise }\end{cases} \\
& B_{g}=\left\{\text { any } i \in G_{\text {on }} \text {, where } \gamma_{i}^{\text {ex ante }} \neq 0\right\} \\
& T_{i}=\left\{\text { any } t \in C_{R}\right. \text {, where } \\
& \left.R C P_{t, i}^{e x} \text { ante }>o_{t, i} \text { and } R_{t, i}^{S E}>0\right\} .
\end{aligned}
$$

$o c_{i}^{\text {reserve }}$ is the cheapest positive marginal opportunity cost of reserve of all types of reserves that have nonzero real-time reserve designation for a resource with a binding capacity constraint. This assumes that a rational resource will trade off energy with 
the reserve with the lowest profit. The bid price for the dispatchable load $j$ is

$$
b_{j}^{\text {ex post }}= \begin{cases}\max \left(L M P_{j}^{e x}\right. \text { ante } & \\ \left.b_{j}^{S E}+o c_{j}^{\text {reserve }}\right), & \text { if } j \in F_{d} \\ 0, & \text { if } j \notin F_{d}\end{cases}
$$

where

$$
\begin{aligned}
& o c_{j}^{\text {reserve }}= \begin{cases}\min _{t \in T_{j}}\left(R C P_{t, j}^{\text {ex ante }}-o_{t, j}\right), & \text { for } i \in B_{d} \\
0, & \text { otherwise }\end{cases} \\
& B_{d}=\left\{\text { any } j \text {, where } \gamma_{j}^{\text {ex ante }} \neq 0\right\} \\
& T_{j}=\left\{\text { any } t \in C_{R} \text {, where } R C P_{t, j}^{e x} \text { ante }>o_{t, j}\right. \\
& \text { and } \left.R_{t, j}^{S E}>0\right\} \text {. }
\end{aligned}
$$

To ensure that the reserve with highest profit be designated first, the ex post reserve quantities or designations for a dispatchable unit $i$ are calculated as

$$
\begin{aligned}
R_{t, i}^{S E}= & \min \left[R_{t, i}^{e x}\right. \text { ante } \\
& \left.\max \left(P_{i}^{\max }-P_{i}^{S E}-\sum_{x \in H_{t, i}} R_{x, i}^{S E}, 0\right)\right]
\end{aligned}
$$

where

$H_{t, i}=\left\{\right.$ any $x \in C_{R}$, where

$$
\left.R C P_{x, i}^{e x} \text { ante }-o_{x, i}>R C P_{t, i}^{e x} \text { ante }-o_{t, i}\right\} .
$$

Similarly, the ex post reserve quantities or designations for a dispatchable load $j$ are

$$
\begin{aligned}
R_{t, j}^{S E}= & \min \left[R_{t, j}^{e x}\right. \text { ante } \\
& \left.\max \left(L_{j}^{S E}-L_{j}^{\min }-\sum_{x \in H_{t, j}} R_{x, j}^{S E}, 0\right)\right]
\end{aligned}
$$

where

$$
\begin{aligned}
H_{t, j}=\left\{\text { any } x \in C_{R},\right. \text { where } \\
\\
\left.\qquad C P_{x, j}^{e x} \text { ante }-o_{x, j}>R C P_{t, j}^{e x} \text { ante }-o_{t, j}\right\} .
\end{aligned}
$$

The formulation of ex post energy price for each location $n$ will be the same as that from the ex ante problem

$$
\begin{aligned}
& L M P_{n}^{\text {ex post }}=\lambda^{\text {ex post }} \cdot\left(1+\frac{\partial P_{\text {loss }}}{\partial D_{n}}\right) \\
& \quad-\sum_{k}\left[\mu_{k}^{\text {ex post }} \cdot \frac{\partial \text { flow }_{k}}{\partial D_{n}}\right]+\sum_{l}\left[\alpha_{l}^{\text {energy }_{l}} \cdot \frac{\partial \mathrm{Iflow}_{l}}{\partial D_{n}}\right] .
\end{aligned}
$$

The decision variables of this optimization problem are $P_{i}$, $L_{j}$, and $S_{l}$. However, it is the ex post LMP ( $L M P_{n}^{e x}$ post) and the reserve MW quantities $\left(R_{t, m}^{S E}\right)$ that are the goal of the ex post energy pricing problem.

\section{B. Ex Post Reserve Pricing}

Like the ex post pricing for energy, the ex post pricing for reserve should also use a similar ex ante formulation to price reserves. As the ex ante analysis shows, the reserve clearing prices are determined by resources' marginal opportunity cost of energy and reserve offer or by the penalty of the reserve deficit. Therefore, pricing reserves through the ex ante formulation (2.2) with resource output fixed at the actual metered point should take into account the effect of the opportunity cost.

After the ex post energy pricing run, ex post energy prices are obtained, and the marginal opportunity cost of energy for each individual resource can be calculated.

1) According to the analysis of the ex ante dispatch problem, a unit with full capacity cleared will reduce one MW of energy in order to produce one more $\mathrm{MW}$ of reserve, resulting in the loss of energy profit, and therefore, the cost to provide additional MW of reserve for this unit is its marginal opportunity cost of energy, which equals to its ex post energy price minus its offer price, plus its reserve offer price. For the unit with capacity cleared partially, the additional cost of providing reserve is zero, since providing additional MW of reserve does not require reducing its generation output.

2) Similar to that of a generator, the marginal opportunity cost of energy for a dispatchable load can be calculated in the same way.

3) In the formulation of the locational reserve constraint (1.3), the local contingency is satisfied by the reserve from the local resources and the unused capacity of the tie line. Compared to local resources, the tie line can be treated as a local generator that produces both energy with the value of tie line flow and reserve with the value of unused tie-line capacity. Therefore, the tie line can be considered as a local reserve supplier, whose cost of reserve is the shadow price of the tie line flow.

With these observations in mind, we can construct the ex post reserve pricing problem as follows:

$$
\begin{aligned}
& \min _{R, S} \sum_{m=i . j}\left[\sum_{t \in C_{R}}\left(c_{t, m}^{\text {ex post }} \cdot R_{t, m}\right)\right]+\sum_{l}\left(o c_{l}^{\text {energy }} \cdot R_{l}\right) \\
& \quad+\sum_{l}\left(c_{l}^{l r} \cdot S_{l}^{l r}\right)+\sum_{s}\left(c_{s}^{s r} \cdot S_{s}^{s r}\right)
\end{aligned}
$$

S.T.

1) Local Reserve Constraints

$$
\left(\alpha_{l}^{\text {reserve }} \geq 0\right): \sum_{m=i, j} \sum_{t \in C_{R}}\left(\delta_{t, m}^{l} \cdot R_{t, m}\right)+R_{l}+S_{l}^{l r} \geq Q_{l}^{S E}
$$

for any ex ante binding constraint $l$.

\section{2) System-Wide Reserve Constraints}

$$
\begin{aligned}
\left(\beta_{s}^{\text {ex post }} \geq 0\right): \sum_{m=i, j} \sum_{t \in C_{R}}\left(\delta_{t, m}^{s} \cdot R_{t, m}\right)+S_{s}^{s r} \geq Q_{s}^{S E} \\
\quad \text { for any ex ante binding constraint } s .
\end{aligned}
$$


3) 10-min Capacity Constraints

$$
\sum_{t \in C_{R}}\left(\delta_{t, m}^{10} \cdot R_{t, m}\right) \leq \sum_{t \in C_{R}}\left(\delta_{t, m}^{10} \cdot R_{t, m}^{S E}\right), \text { for any } m
$$

4) 30-min Capacity Constraints

$$
\sum_{t \in C_{R}}\left(R_{t, m}\right) \leq \sum_{t \in C_{R}}\left(R_{t, m}^{S E}\right), \text { for any } m .
$$

5) Boundary Constraints for Reserves

$$
\begin{aligned}
& \left\{\begin{array}{l}
R_{t, m} \geq 0 \quad \text { for any } t \text { if } m \in F_{r} \\
R_{t, m}=R_{t . m}^{S E}, \quad \text { for any } t \text { if } m \notin F_{r}
\end{array} \text { for any } m\right. \\
& 0 \leq R_{l} \leq R_{l}^{S E} \text { for each binding local } \\
& \quad \text { reserve constraint } l \\
& S_{l}^{l r} \geq 0 \\
& S_{s}^{s r} \geq 0
\end{aligned}
$$

where

$F_{r} \quad$ set of flexible reserve resources;

$R_{l} \quad$ reserve from the unused capacity of the tie line to the local area $l$, and $R_{l}^{S E}=$ Iflow $_{l}^{\max }-$ Iflow $_{l}^{S E}$.

In the ex post pricing, the actual reserve requirement should be the real-time available amount since this ex post pricing is not a reserve dispatch. The requirements are

$$
\begin{aligned}
Q_{l}^{S E}= & \sum_{m=i, j} \sum_{t \in C_{R}}\left(\delta_{t, m}^{l} \cdot R_{t, m}^{S E}\right)+R_{l}^{S E} \\
& +S_{l}^{l r, \text { ex ante }}-\Delta \\
Q_{s}^{S E}= & \sum_{m=i, j} \sum_{t \in C_{R}}\left(\delta_{t, m}^{s} \cdot R_{t, m}^{S E}\right)+S_{s}^{\text {sr,ex ante }}-\Delta .
\end{aligned}
$$

The small perturbation $\Delta$ in (9.1) and (9.2) is used to avoid dual degeneracy.

The ex post reserve offer price for resource $m$ is

$$
c_{t, m}^{\text {ex post }}=\left\{\begin{array}{cl}
\min \left(R C P_{t, m}^{\text {ex ante }}\right. & \\
\left.o c_{m}^{\text {energy }}+o_{t, m}\right), & \text { if } m \in F_{r} \\
0, & \text { if } m \notin F_{r}
\end{array}\right.
$$

and its marginal opportunity cost of energy is

$o c_{m}^{\text {energy }}= \begin{cases}\max \left(L M P_{m}^{\text {ex post }}-c_{m}^{S E}, 0,\right) & \text { if } m \in B_{c} \\ 0, & \text { otherwise }\end{cases}$ for any $m$

where

$$
\begin{aligned}
& c_{m}^{S E}= \\
& \left\{\begin{array}{l}
c_{i}^{S E} \text { for any } m=i \\
b_{j}^{S E} \text { for any } m=j
\end{array}\right. \\
& B_{c}=B_{g} \cup B_{d}
\end{aligned}
$$

price corresponding to the actual generation or load in the offer or bid curve; set of all reserve resources that have binding capacity constraint in the ex ante dispatch.

The marginal opportunity cost of energy for the tie line $o c_{l}^{\text {energy }}$ is the shadow price of the local reserve constraint $\left(\alpha_{l}^{\text {energy }}\right)$ in the ex post pricing problem for energy.
The ex post reserve price is

$$
\begin{aligned}
R C P_{t, m}^{\text {ex post }}= & \sum_{l}\left[\alpha_{l}^{\text {reserve }} \cdot \delta_{t, m}^{l}\right] \\
& +\sum_{s}\left[\beta_{s}^{\text {ex post }} \cdot \delta_{t, m}^{s}\right] .
\end{aligned}
$$

The rules that determine the flexibility $\left(F_{r}\right)$ of a reserve resource should be carefully designed. For simple implementation, we can use the definition of $F_{g}$ and $F_{d}$. Under this reserve pricing, in general, the reserve price is set by the resource with the highest ex post offer price $c_{t, m}^{\text {ex post }}$. Each resource's ex post reserve offer price is lower than the ex ante reserve clearing price; the ex post reserve clearing prices tend to be less than or equal to the ex ante clearing prices. If the ex ante marginal reserve resource is somehow set to be inflexible, the ex post reserve clearing price will be lower than the ex ante reserve clearing price. Therefore, the unit that over-performs in the energy market could be penalized by the reduced reserve price.

The decision variables of this optimization problem are $R_{t, m}, R_{l}, S_{l}$, and $S_{s}$. However, it is the ex post reserve price $\left(R C P_{t, m}^{e x}{ }^{\text {post }}\right)$ rather than the solution of these decision variables that is the major goal of the ex post reserve pricing problem.

\section{Discussion of the Proposed Pricing Schema}

The procedure described above does not calculate the energy and reserve prices in a joint optimization; however, the decoupled pricing for energy and reserve with the marginal opportunity cost indeed respects the coupling effect between them. Therefore, the ex post prices generated by this procedure can be reasonably justified according to the ex post pricing principles. As shown in the above discussion, the advantages of this approach are as follows.

1) It decouples the pricing of energy from that of reserve and makes the pricing of energy and reserve much easier. The decoupling of energy and reserve also avoids defining complex pricing rules according to the binding statuses of unit level constraints in the co-optimization.

2) The approach significantly simplifies the implementation of the ex post energy and reserve pricing rules independently while taking into account the coupling effect between energy and reserves.

Same as any other pricing model, this proposed method is based on certain assumptions. In the following, we will discuss these assumptions and their impact on the pricing model.

1) The ex ante reserve clearing prices are used to estimate the real-time energy bid prices, when we price energy ex post. Since the ex ante reserve clearing price may not be the same as the ex post reserve clearing price, we may iterate between these two pricing problems to find the final solution. However, this is not necessary, since the prices produced by the first iteration could be explained as one set of rational prices.

2) The ex post pricing for energy uses the values of $\Delta_{u p}$ and $\Delta_{d n}$ to simulate the pricing effect caused by ramp constraints in the ex ante dispatch problem. In practice, set- 


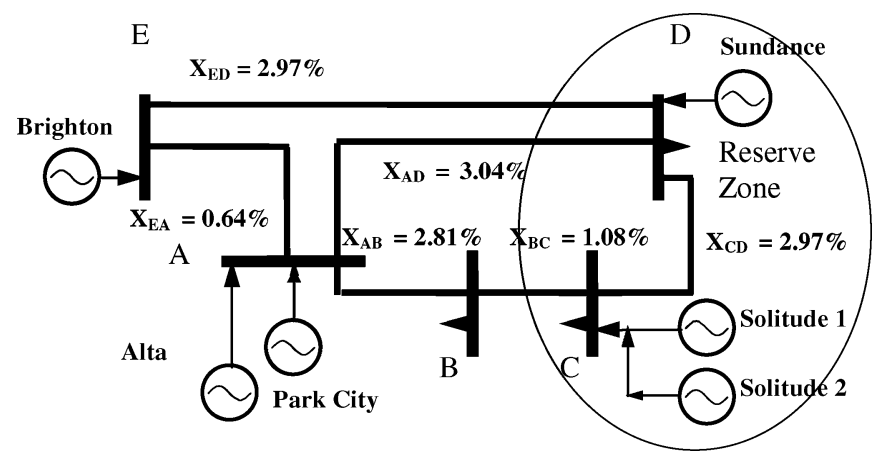

Fig. 2. Five-bus system diagram.

TABLE I

BUS LEVEL INFORMATION FOR ALL SCENARIOS

\begin{tabular}{c|r|r|r}
\hline \hline Bus ID & $\begin{array}{c}\text { Bus } \\
\text { Load }\end{array}$ & LF & GSF \\
\hline A & 0 & 0.0466 & 0.5848 \\
\hline B & 350 & -0.0094 & 0.5945 \\
\hline C & 250 & -0.0031 & -0.4098 \\
\hline D & 250 & 0.0163 & -0.4225 \\
\hline E & 0 & 0.0596 & 0.5800 \\
\hline \hline
\end{tabular}

ting the value of $\Delta_{u p}$ to be relatively much smaller than the value of $\Delta_{d n}$ and capping the energy offer price by the ex ante energy prices do, in most cases, prevent ramp constrained unit from setting the ex post energy prices. However, the values of $\Delta_{u p}$ and $\Delta_{d n}$ in theory influence the ex post prices.

3) Like any other ex post pricing schema, it is dependent on the formulation of the ex ante dispatch. A modification is necessary upon the change of the dispatch problem. For instance, when the down-reserve requirements are considered in the ex ante dispatch, the ex post energy offer price of a unit has to be reduced by the as-bid reserve profit, and the ex post reserve pricing has to include down-reserve products. However, the modification is not substantial.

\section{NUMERICAL EXAMPLE}

In this section, we will perform studies of ex post pricing on a five-bus system based on different ex ante dispatch scenarios. The system information is shown in Fig. 2. The reactance (X) shown in Fig. 2 is in per unit value.

The bus level information is listed in Table I. LF is the loss factor at each bus $\left(\partial P_{\text {loss }} / \partial P_{i}\right)$, and GSF is the generation shift factor $\left(\partial \mathrm{Iflow}_{l} / \partial P_{i}\right)$ of a closed interface that contains lines A-D, E-D, and B-C. These factors are calculated from a base-case power flow. The flow limit for the interface is $200 \mathrm{MW}$, and the local reserve requirement for the reserve zone (buses $\mathrm{C}$ and $\mathrm{D}$ ) is $300 \mathrm{MW}$. The system-wide 10-min spinning, 10-min non-spinning, and 30-min operating reserve requirements are 200, 200, and $300 \mathrm{MW}$, respectively. (In the dispatch, the system-wide reserve constraints are enforced on 10-min spin $200 \mathrm{MW}, 10-\mathrm{min}$ total reserve $400 \mathrm{MW}$, and total 30-min reserve $700 \mathrm{MW}$.) In addition, the penalty prices for all of the reserve constraints, including the local reserve constraint, are set to $\$ 1000$. The system losses are linearized around a predetermined operating point; the linearization error is $-15 \mathrm{MW}$ (please see [15] for detailed implementation of marginal losses); and the dispatch interval is $15 \mathrm{~min}$ in the ex ante dispatch.

Based on the above information, three different scenarios are designed to study the ex post pricing model. In each of the following cases, the load at each bus, the loss factors, and the generation shift factors are kept constant in both the ex ante dispatch and the ex post pricing. For simplicity, we assume the reserve offer prices are all zero for all cases.

Case 1: In this scenario, the system-wide total 10-min reserve and total 30-min reserve constraints are binding. The generators' characteristics and ex ante dispatch results are presented in Table II. Note that in Table II, "Pmin" is the minimum generation output $P_{i}^{\text {min }}$; "Pmax" is the maximum generation level $P_{i}^{\max }$; "SE MW" is the generator's initial output level in the ex ante dispatch; "LMP" is the ex ante clearing price for energy; and "RCP" is the ex ante clearing price for reserve. All the quantities in Tables II-VII are in MWs, and all the prices are in $\$ / \mathrm{MW}$

The coupling effect of energy and reserve for each individual unit is shown in "Capacity Binding Status" $\left(B_{g}\right)$ of Table II, which indicates that Alta, Brighton, Solitude 1, and Solitude 2 incur the loss of opportunity cost. Solitude 1 and Solitude 2 have lower marginal opportunity cost of energy (the difference between ex ante LMP and the offer price), so they are backed down to their minimum output level; ParkCity is upward ramp constrained; Sundance is the marginal unit and sets the energy price; Brighton is the marginal unit whose marginal opportunity cost of energy (133.85-20 or \$113.85) sets the ex ante spinning and non-spinning reserve prices; Alta is also a marginal unit, and its marginal opportunity cost of energy (135.69-25 or \$110.69) becomes the ex ante clearing price of the operating reserve.

Based on this ex ante dispatch scenario, we can examine how the ex post pricing schema behaves when one of the marginal units over-performs. As discussed above, unit Alta is a marginal unit, and its marginal opportunity cost is the same as the ex ante clearing price of operating reserve. In the ex post pricing, we assume unit Alta over-performs and generates at $80 \mathrm{MW}$, which is $10 \mathrm{MW}$ above its ex ante dispatch MW, while unit Brighton generates $350 \mathrm{MW}$. All other units perform consistently at their ex ante dispatch.

Given the ex ante capacity binding status, and units' actual performance ("Ex Post Energy SE MW"), the ex post energy pricing results are shown in Table III. Note that the ex post energy SE MW $\left(P_{i}^{S E}\right)$ is not the solution of the pricing problem. We list it here since it is the actual amount of energy for which the generator will be paid. The flexibility status $\left(F_{g}\right)$ and the "Energy Offer Price" $\left(c_{i}^{\text {ex post }}\right)$ are determined by comparing the ex ante dispatch MW with SE MW. Since the ex ante marginal unit (Sundance) follows the dispatch instruction and generates at $126.28 \mathrm{MW}$, ex post LMPs are the same as ex ante LMPs.

The ex post reserve quantities $\left(R_{t, i}^{S E}\right)$ shown in Table III under columns "SE sp, nsp or op MW" are calculated based on units' actual performance and their ex ante reserve dispatch from ex post energy pricing. We listed them under the results of reserve 
TABLE II

EX ANTE Dispatch Results For CASE 1

\begin{tabular}{|c|c|c|c|c|c|c|c|c|c|c|c|c|c|c|}
\hline \multirow[b]{2}{*}{$\begin{array}{c}\text { Generator } \\
\text { Name }\end{array}$} & \multicolumn{5}{|c|}{ Units' Characteristics } & \multicolumn{4}{|c|}{ Ex Ante Dispatch MWs } & \multicolumn{4}{|c|}{ Ex Ante Clearing Prices (\$/MW) } & \multirow[b]{2}{*}{$\begin{array}{c}\text { Capacity } \\
\text { Binding } \\
\text { Status }\end{array}$} \\
\hline & $\begin{array}{c}\text { Offer } \\
\text { Price } \\
(\$ / \mathrm{MW})\end{array}$ & $\begin{array}{l}\text { Pmin } \\
(\mathrm{MW})\end{array}$ & $\begin{array}{l}\text { Pmax } \\
(\mathrm{MW})\end{array}$ & $\begin{array}{l}\text { Ramp Rate } \\
\text { (MW/Min) }\end{array}$ & $\begin{array}{c}\text { SE } \\
\text { MW }\end{array}$ & GEN & SP & NSP & OP & LMP & RCP SP & $\begin{array}{l}\text { RCP } \\
\text { NSP }\end{array}$ & $\mathrm{RCP} O \mathrm{OP}$ & \\
\hline Alta & 25 & 0 & 250 & 10 & 50 & 70.00 & 100.00 & 0.00 & 80.00 & 135.69 & 113.85 & 113.85 & 110.69 & Yes \\
\hline ParkCity & 30 & 50 & 250 & 4 & 50 & 110.00 & 40.00 & 0.00 & 80.00 & 135.69 & 113.85 & 113.85 & 110.69 & No \\
\hline Brighton & 20 & 300 & 400 & 10 & 400 & 360.00 & 40.00 & 0.00 & 0.00 & 133.85 & 113.85 & 113.85 & 110.69 & Yes \\
\hline Solitude 1 & 60 & 200 & 300 & 10 & 200 & 200.00 & 100.00 & 0.00 & 0.00 & 142.77 & 113.85 & 113.85 & 110.69 & Yes \\
\hline Solitude 2 & 50 & 0 & 200 & 10 & 50 & 0.00 & 100.00 & 0.00 & 100.00 & 142.77 & 113.85 & 113.85 & 110.69 & Yes \\
\hline Sundance & 140 & 100 & 200 & 2 & 100 & 126.28 & 20.00 & 0.00 & 40.00 & 140.00 & 113.85 & 113.85 & 110.69 & No \\
\hline
\end{tabular}

TABLE III

Ex Post ENERgy AND Reserve PRICING Results for CASE 1

\begin{tabular}{|c|c|c|c|c|c|c|c|c|c|c|c|c|c|}
\hline \multirow{3}{*}{$\begin{array}{l}\text { Generator } \\
\text { Name }\end{array}$} & \multicolumn{4}{|c|}{ Ex Post Energy Pricing Results } & \multicolumn{9}{|c|}{ Ex Post Reserve Pricing Results } \\
\hline & \multirow{2}{*}{$\begin{array}{c}\text { Ex Post } \\
\text { Energy } \\
\text { Offer }\end{array}$} & \multirow[b]{2}{*}{$\begin{array}{c}\text { Flexibility } \\
\text { Status }\end{array}$} & \multirow{2}{*}{\begin{tabular}{|c|} 
Ex Post \\
Energy SE \\
MW
\end{tabular}} & \multirow[b]{2}{*}{$\begin{array}{c}\text { Ex Post } \\
\text { LMP }\end{array}$} & \multicolumn{3}{|c|}{ Ex Post Reserve Offer Price } & \multicolumn{3}{|c|}{ Ex Post Reserve MW } & \multicolumn{3}{|c|}{ Reserve Clearing Price } \\
\hline & & & & & SP & NSP & $\mathrm{OP}$ & $\begin{array}{c}\text { SE SP } \\
\text { MW }\end{array}$ & $\begin{array}{c}\text { SE NSP } \\
\text { MW }\end{array}$ & $\begin{array}{c}\text { SE OP } \\
\text { MW }\end{array}$ & RCP SP & $\begin{array}{l}\text { RCP } \\
\text { NSP }\end{array}$ & $\mathrm{RCP}$ \\
\hline Ita & 0.00 & & 80.00 & 135.69 & 0.00 & 0.00 & 0.00 & 100.00 & 0.00 & 70.00 & 113.85 & 113.85 & \\
\hline arkCity & 30.00 & Yes & 110.00 & & 0.00 & 0.00 & 0.00 & 40.00 & 0.00 & 80.00 & 113.85 & 113.85 & 92.77 \\
\hline righ & 3.85 & $\overline{\mathrm{Y}}$ & 350.00 & 133.85 & 113.85 & 113.85 & 110.69 & 40.00 & 0.00 & 0.00 & 113.85 & 113.85 & 92.7 \\
\hline lit & 2.77 & Yes & 200.00 & 1 & 2.77 & 2.77 & 82.77 & 100.00 & 0.00 & 0.00 & 113.85 & 113.85 & 92.7 \\
\hline 74 & 2.77 & & 0.00 & & 92.77 & 92.77 & 92.77 & 100.00 & 0.00 & 100.00 & & 113.85 & 92.7 \\
\hline undance & 140.00 & Yes & 126.28 & 140.00 & 0.00 & 0.00 & 0.00 & 20.00 & 0.00 & 40.00 & 113.85 & 113.85 & 92.7 \\
\hline
\end{tabular}

TABLE IV

Ex ANTE Dispatch Results for CASE 2

\begin{tabular}{|c|c|c|c|c|c|c|c|c|c|c|c|c|c|c|}
\hline \multirow[b]{2}{*}{$\begin{array}{c}\text { Generator } \\
\text { Name }\end{array}$} & \multicolumn{5}{|c|}{ Units' Characteristics } & \multicolumn{4}{|c|}{ Ex Ante Dispatch MWs } & \multicolumn{4}{|c|}{ Ex Ante Clearing Prices $(\$ / \mathrm{MW})$} & \multirow[b]{2}{*}{$\begin{array}{c}\text { Capacity } \\
\text { Binding } \\
\text { Status }\end{array}$} \\
\hline & $\begin{array}{c}\text { Offer } \\
\text { Price } \\
(\$ / \mathrm{MW}) \\
\end{array}$ & $\begin{array}{l}\text { Pmin } \\
(\mathrm{MW})\end{array}$ & $\begin{array}{l}\text { Pmax } \\
(M W)\end{array}$ & $\begin{array}{l}\text { Ramp Rate } \\
\text { (MW/Min) }\end{array}$ & SE MW & GEN & SP & NSP & $\mathrm{OP}$ & LMP & RCP SP & $\begin{array}{l}\text { RCP } \\
\text { NSP }\end{array}$ & $\begin{array}{c}\mathrm{RCP} \\
\mathrm{OP}\end{array}$ & \\
\hline Alta & 25 & 0 & 300 & 20 & 50 & 29.62 & 200.00 & 0.00 & 70.38 & 25.00 & 0.00 & 0.00 & 0.00 & No \\
\hline ParkCity & 30 & 50 & 300 & 20 & 50 & 50.00 & 200.00 & 0.00 & 50.00 & 25.00 & 0.00 & 0.00 & 0.00 & No \\
\hline Brighton & 20 & 200 & 400 & 20 & 400 & 400.00 & 0.00 & 0.00 & 0.00 & 24.31 & 0.00 & 0.00 & 0.00 & No \\
\hline Solitude 1 & 60 & 200 & 300 & 4 & 200 & 200.00 & 40.00 & 0.00 & 60.00 & 140.42 & 111.33 & 111.33 & 111.33 & Yes \\
\hline Solitude 2 & 50 & 0 & 200 & 2 & 50 & 80.00 & 20.00 & 0.00 & 40.00 & 140.42 & 111.33 & 111.33 & 111.33 & No \\
\hline Sundance & 140 & 100 & 200 & 2 & 100 & 103.74 & 20.00 & 0.00 & 40.00 & 140.00 & 111.33 & 111.33 & 111.33 & No \\
\hline
\end{tabular}

pricing just to make the analysis easier; they are by no means the reserve quantity solution of the ex post reserve pricing problem. Table III shows that Alta's OP reserve MW is reduced by 10 MW due to its over-performance in the energy market. Given the same reserve flexibility rule as that of the energy pricing, ex post reserve offer prices for all categories are computed based on the ex post energy prices (LMPs) and are presented in Table III under reserve pricing results, which also shows the ex post reserve clearing prices. Compared with the ex ante price, the ex post operating reserve price is $\$ 92.77$ rather than its ex ante value $\$ 110.69$. Since Alta over-performs and tends not to respond to the dispatch instruction, to satisfy the operating reserve requirement, the operating reserve from Solitude 2 becomes marginal. Its corresponding ex post reserve offer price $\$ 92.77$ or the marginal opportunity cost of energy sets the ex post operating reserve price. Since the marginal energy opportunity cost of every unit that provides operating reserve is lower than the ex post operating reserve prices, all units are satisfied, except for the non-performing unit Alta, which is penalized. Thus, this set of ex post prices is rational.
Case 2: In this scenario, the local reserve constraint is binding such that the reserve price separation occurs. The generators' characteristics and the ex ante dispatch results are shown in Table IV. In this case, Alta and Sundance are the marginal units that determine the ex ante prices. The shadow price of the local reserve constraint becomes the ex ante local reserve clearing price. This is because units outside the local reserve zone have to be dispatched down to make more room on the tie line, in order to serve an increment of reserve to the local reserve zone. Such a re-dispatch cost becomes the ex ante reserve clearing price and introduces congestion in the energy price. In addition, the energy-reserve coupling effect exists for Solitude 1, and this unit is dispatched to its minimum.

In the ex post pricing, we assume that units Alta and ParkCity do not follow the dispatch instructions and over-generate 10 MW each, while unit Brighton is backed down by $20 \mathrm{MW}$. We would expect Brighton and Sundance to set the ex post prices. The ex post energy pricing results are shown in Table V. Since the ex ante marginal unit Alta fails to follow dispatch, it is ineligible to set the ex post price. Therefore, Brighton and Solitude 1 
TABLE V

Ex Post ENERGY AND RESERVE PRICING RESULTS FOR CASE 2

\begin{tabular}{|c|c|c|c|c|c|c|c|c|c|c|c|c|c|}
\hline \multirow{3}{*}{$\begin{array}{c}\text { Generator } \\
\text { Name }\end{array}$} & \multicolumn{4}{|c|}{ Ex Post Energy Pricing Results } & \multicolumn{9}{|c|}{ Ex Post Reserve Pricing Results } \\
\hline & \multirow{2}{*}{$\begin{array}{c}\text { Ex Post } \\
\text { Energy } \\
\text { Offer }\end{array}$} & \multirow{2}{*}{$\begin{array}{c}\text { Flexibility } \\
\text { Status }\end{array}$} & \multirow{2}{*}{$\begin{array}{c}\text { Ex Post } \\
\text { Energy SE } \\
\text { MW }\end{array}$} & \multirow{2}{*}{$\begin{array}{c}\text { Ex Post } \\
\text { LMP }\end{array}$} & \multicolumn{3}{|c|}{ Ex post Reserve Offer Price } & \multicolumn{3}{|c|}{ Ex Post Reserve MW } & \multicolumn{3}{|c|}{ Reserve Clearing Price } \\
\hline & & & & & SP & NSP & OP & $\begin{array}{l}\text { SE SP } \\
\text { MW }\end{array}$ & \begin{tabular}{|c|} 
SE NSP \\
MW
\end{tabular} & $\begin{array}{c}\text { SE OP } \\
\text { MW }\end{array}$ & RCP SP & $\begin{array}{l}\text { RCP } \\
\text { NSP }\end{array}$ & $\mathrm{RC}$ \\
\hline Alta & 0 & No & 39.62 & 20.65 & 0.00 & 0.00 & 0.00 & 200.00 & 0.00 & 60.38 & 0.00 & 0.00 & 0.00 \\
\hline ParkCity & 0 & No & 60.00 & 20.65 & 0.00 & 0.00 & 0.00 & 200.00 & 0.00 & 40.00 & 0.00 & 0.00 & 0.00 \\
\hline Brighton & 20 & Yes & 380.00 & 20.00 & 0.00 & 0.00 & 0.00 & 0.00 & 0.00 & 0.00 & 0.00 & 0.00 & 0.00 \\
\hline Solitude 1 & 140.42 & Yes & 200.00 & 140.42 & 80.42 & 80.42 & 80.42 & 40.00 & 0.00 & 60.00 & 115.80 & 115.80 & 115.80 \\
\hline Solitude 2 & 50 & Yes & 80.00 & 140.42 & 0.00 & 0.00 & 0.00 & 20.00 & 0.00 & 40.00 & 115.80 & 115.80 & 115.80 \\
\hline Sundance & 140 & Yes & \begin{tabular}{|l|}
103.74 \\
\end{tabular} & 140.09 & 0.00 & 0.00 & 0.00 & 20.00 & 0.00 & 40.00 & 115.80 & 115.80 & 115.80 \\
\hline
\end{tabular}

TABLE VI

EX ANTE DisPaTCH RESUlTS FOR CASE 3

\begin{tabular}{|c|c|c|c|c|c|c|c|c|c|c|c|c|c|c|}
\hline \multirow[b]{2}{*}{$\begin{array}{c}\text { Generator } \\
\text { Name }\end{array}$} & \multicolumn{5}{|c|}{ Units' Characteristics } & \multicolumn{4}{|c|}{ Ex Ante Dispatch MWs } & \multicolumn{4}{|c|}{ Ex Ante Clearing Prices $(\$ / M W)$} & \multirow[b]{2}{*}{$\begin{array}{c}\text { Capacity } \\
\text { Binding } \\
\text { Status }\end{array}$} \\
\hline & $\begin{array}{c}\text { Offer } \\
\text { Price } \\
(\$ / \mathrm{MW})\end{array}$ & $\begin{array}{l}\text { Pmin } \\
\text { (MW) }\end{array}$ & $\begin{array}{l}\text { Pmax } \\
\text { (MW) }\end{array}$ & $\begin{array}{l}\text { Ramp Rate } \\
\text { (MW/Min) }\end{array}$ & SE & GEN & SP & NSP & OP & LMP & SP & $\begin{array}{l}\text { RCP } \\
\text { NSP }\end{array}$ & $\mathrm{RC}$ & \\
\hline Alta & 25 & 0 & 250 & 20 & 50 & 87.36 & 162.64 & 0.00 & 0.00 & 1025.00 & 1000.00 & 1000.00 & 1000.00 & Yes \\
\hline ParkCity & 30 & 50 & 250 & 10 & 50 & 50.00 & 100.00 & 0.00 & 100.00 & 1025.00 & 1000.00 & 1000.00 & 1000.00 & Yes \\
\hline Brighton & 20 & 200 & 400 & 10 & 400 & 250.00 & 100.00 & 0.00 & 50.00 & 1011.09 & 1000.00 & 1000.00 & 1000.00 & Yes \\
\hline e 1 & 60 & 200 & 300 & 4 & 200 & 260.00 & 40.00 & 0.00 & 0.00 & 1078.49 & 1000.00 & 1000.00 & 1000.00 & $\mathrm{Yc}$ \\
\hline & 50 & 0 & 200 & 2 & 50 & 80.00 & 20.00 & 0.0 & 40.00 & 1078.49 & 1000.00 & 1000.00 & & \\
\hline indance & 140 & 100 & 200 & 2 & 100 & 130.00 & 20.00 & \begin{tabular}{l|l}
0.00 \\
\end{tabular} & 40.00 & 1057.57 & \begin{tabular}{|l|}
1000.00 \\
\end{tabular} & 1000.00 & 1000.00 & No \\
\hline
\end{tabular}

TABLE VII

Ex Post ENERGy AND ReSERVE PRICING RESULTS FOR CASE 3

\begin{tabular}{|c|c|c|c|c|c|c|c|c|c|c|c|c|c|}
\hline \multirow{3}{*}{$\begin{array}{c}\text { Generator } \\
\text { Name }\end{array}$} & \multicolumn{4}{|c|}{ Ex Post Energy Pricing Results } & \multicolumn{9}{|c|}{ Ex Post Reserve Pricing Results } \\
\hline & \multirow{2}{*}{$\begin{array}{c}\text { Ex Post } \\
\text { Energy } \\
\text { Offer }\end{array}$} & \multirow{2}{*}{\begin{tabular}{|c|} 
Energy \\
Flexibility \\
Status \\
\end{tabular}} & \multirow{2}{*}{$\begin{array}{l}\text { Ex Post } \\
\text { SE MW }\end{array}$} & \multirow[b]{2}{*}{$\begin{array}{c}\text { Ex Post } \\
\text { LMP }\end{array}$} & \multicolumn{3}{|c|}{ Ex post Reserve Offer Price } & \multicolumn{3}{|c|}{ Ex Post Reserve MW } & \multicolumn{3}{|c|}{ Reserve Clearing Price } \\
\hline & & & & & SP & NSP & OP & $\begin{array}{l}\text { SE SP } \\
\text { MW }\end{array}$ & $\begin{array}{c}\text { SE NSP } \\
\text { MW }\end{array}$ & $\begin{array}{c}\text { SE OP } \\
\text { MW }\end{array}$ & RCP SP & $\begin{array}{l}\text { RCP } \\
\text { NSP }\end{array}$ & RCP C \\
\hline ta & 0 & No & 107.36 & & & 0. & & 142.64 & 0.00 & 0.00 & 1000.00 & 1000.00 & \\
\hline $\mathrm{rkC}$ & 0 & No & & & & 0.0 & & 100.00 & 0.0 & 90.00 & 1000.00 & 1000.00 & 0.0 \\
\hline igl & 11.09 & Y & 0.00 & & 991.09 & 91.09 & 91.09 & 100.00 & 0.0 & 50.00 & 1000.00 & 1000.00 & $1000 .($ \\
\hline Mtic & 1060 & Yes & 260.00 & & 00.00 & 00.00 & 00.00 & 40.00 & 0.0 & 0.00 & 1000.00 & 1000.00 & 1000.0 \\
\hline litude 2 & 50 & Yes & 80.00 & & 0.00 & 0.00 & 0.00 & 20.00 & 0.00 & 40.00 & 1000.00 & 1000.00 & 1000.0 \\
\hline undance & 140 & Yes & 130.00 & 1057.57 & 0.00 & 0.00 & 0.00 & 20.00 & 0.00 & 40.00 & 1000.00 & 1000.00 & 1000.0 \\
\hline
\end{tabular}

units set the ex post energy price. Since some room must be kept on the tie line to satisfy the local reserve needs, the energy flow in the tie line becomes congested. The ex post shadow price of the tie line is now $\$ 115.85$. Compared with ex ante prices, the prices received by Alta and ParkCity are even lower than their offer prices, resulting in reduced energy revenue.

With the given ex post energy prices, and the increased opportunity cost for the tie line $(\$ 115.85)$, the ex post reserve MW quantities and prices are computed and shown in Table $\mathrm{V}$ under the column of "Ex post Reserve Pricing Results." The operating reserve quantities for both Alta and ParkCity are reduced due to nonperformance, while the ex post local reserve clearing price is set by the increased tie-line marginal opportunity cost of energy, due to the increased congestion caused by the over-performance of Alta and ParkCity. Units in the local reserve zone benefit both from energy and reserve markets slightly for their proper behavior. In short, both ex post energy and reserve prices are reasonable.

Case 3: In this scenario, the system-wide operating reserve constraint is violated, which is caused by a capacity shortage.
The generators' characteristics and ex ante dispatch results are shown in Table VI, which indicates that the reserve constraint penalty $(\$ 1000)$ and the offer price of Alta unit (\$25) jointly determine the ex ante clearing prices. Due to the reserve scarcity condition, the ex ante reserve clearing prices become $\$ 1000$.

In the ex post pricing, we assume that units Alta and ParkCity do not follow the dispatch instructions and over-generate 20 and $10 \mathrm{MW}$, respectively, while Brighton is backed down by $30 \mathrm{MW}$. We would expect Brighton to set the ex post prices. The ex post energy and reserve pricing results are both shown in Table VII.

Because units Alta and ParkCity fail to follow dispatch, they are ineligible to set the ex post energy and reserve prices. As shown in Table VII, Brighton sets the ex post energy price with its ex ante energy price or LMP (\$1011.09), resulting in the same prices as ex ante for all units. Those ex post energy prices are reasonable, because prices for Alta and ParkCity would have been even higher than their ex ante prices, had Brighton set the ex post price with the uncapped offer price $(\$ 20)$ plus the reserve penalty $(\$ 1000)$. 
Same as the ex ante dispatch, the ex post reserve pricing sets the ex post reserve clearing prices by the reserve penalty. However, Table VII shows that Alta and ParkCity will receive less payment for reserves due to the fact that their ex post reserve quantities are reduced by 20 and $10 \mathrm{MW}$, respectively. Therefore, the ex post prices are justifiable, even though the over-performed units Alta and ParkCity are not penalized by ex post prices.

\section{CONCLUSION}

This paper presents an ex post pricing schema based on a design of the co-optimized real-time dispatch. In the proposed approach, ex post energy and reserve prices are calculated in a decoupled way: the ex post energy prices are calculated first with the consideration of ex ante marginal opportunity cost of reserve that is a function of the ex ante clearing prices for reserve products; then, the ex post reserve prices for all locations are computed, using the ex post marginal opportunity cost of energy that is determined by the ex post energy prices. This approach not only simplifies the ex post pricing rules without considering the different constraints binding statuses but also reduces the uncertainty caused by heuristics of the co-optimization approach in the pricing of energy and reserve products without ignoring the coupling effect between them. Since the ex post pricing is based on the analysis of the ex ante dispatch and the pricing rules, this proposed schema, like any other ex post pricing model, is subject to the change of dispatch model and market rules. However, the concept of including the marginal opportunity cost in the energy and reserve pricing still applies.

\section{REFERENCES}

[1] F. C. Schweppe, M. C. Caramanis, R. D. Tabors, and R. E. Robn, Spot Pricing of Electricity. Norwell, MA: Kluwer, 1998.

[2] Remedying Undue Discrimination Through Open Access Transmission Service and Standard Market Design, Notice of Proposed Rulemaking, IV FERC Stats. and Regs. 32 563, Docket No. RM01-12-000.

[3] Transmission and Dispatching Operations. [Online]. Available: http:// www.nyiso.com/services/documents/manuals/index.html.
[4] PJM Manual 11: Scheduling Operations. [Online]. Available: http:// www.pjm.com/contributions/pjm-manuals/pdf/m11v23.pdf.

[5] New England Power Pool-Market Rule 1, ch. 2. [Online]. Available: http://www.iso-ne.com.

[6] J. Chen, J. S. Thorp, R. J. Thomas, and T. D. Mount, "Locational pricing and scheduling for an integrated energy-reserve market," in Proc. 36th Hawaii Int. Conf. System Sciences, 2003, pp. 54-63.

[7] X. Ma, D. Sun, and K. Cheung, "Energy and reserve dispatch in a multizone electricity market," IEEE Trans. Power Syst., vol. 14, no. 3, pp. 913-919, Aug. 1999.

[8] T. Wu, M. Rothleder, Z. Alaywan, and A. D. Papalexopoulos, "Pricing energy and ancillary services in integrated market systems by an optimal power flow," IEEE Trans. Power Syst., vol. 19, no. 1, pp. 339-347, Feb. 2004.

[9] K. Cheung, P. Shamsollahi, D. Sun, J. Milligan, and M. Potishnak, "Energy and ancillary service dispatch for the interim ISO New England electricity market," IEEE Trans. Power Syst., vol. 15, no. 3, pp. 968-974, Aug. 2000.

[10] D. Gan and E. Litvinov, "Energy and reserve market designs with explicit consideration to lost opportunity cost," IEEE Trans. Power Syst., vol. 18, no. 1, pp. 53-59, Feb. 2003.

[11] W. Hogan, Contract Networks for Electric Power Transmission: Technical Reference. [Online]. Available: http://ksghome.harvard.edu/ .whogan.cbg.Ksg/.

[12] J. Ring, "Dispatch based pricing in decentralized power systems," Ph.D. dissertation, Dept. Management, Univ. Canterbury, Canterbury, U.K., 1995.

[13] A. Ott, "Experience with PJM market operation, system design, and implementation," IEEE Trans. Power Syst., vol. 18, no. 2, pp. 528-534, May 2003.

[14] Ancillary Service Market Enhancements White Paper. [Online]. Available: http://www.iso-ne.com.

[15] E. Litvinov, T. Zheng, G. Rosenwald, and P. Shamsollahi, "Marginal loss modeling in LMP calculation," IEEE Trans. Power Syst., vol. 19, no. 2, pp. 880-888, May 2004.

Tongxin Zheng (M'02) received the Ph.D degree in electrical engineering from Clemson University, Clemson, SC, in 1999.

He is currently a Principal Analyst at ISO New England, Holyoke, MA. His main interests are power system optimization, and electricity market design.

Eugene Litvinov (M'91) received the B.S. and M.S. degrees in 1973 from the Technical University, Kiev, Ukraine, and the Ph.D. degree in 1987 from Urals Polytechnic Institute, Sverdlovsk, Russia.

He is currently a Director of Technology at the ISO New England, Holyoke, MA. His main interests are power system market clearing models, system security, computer applications to power systems, and information technology. 